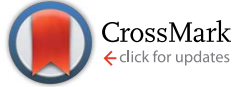

Cite this: Soft Matter, 2015, 11, 297

Received 15th October 2014

Accepted 3rd November 2014

DOI: $10.1039 / c 4 s m 02273 d$

www.rsc.org/softmatter

\section{Polydispersity and gelation in concentrated colloids with competing interactions $\uparrow$}

\author{
Tian Hui Zhang, ${ }^{\text {abc }}$ Bonny W. M. Kuipers, ${ }^{\text {b }}$ Wen-de Tian, ${ }^{\text {ac }}$ Jan Groenewold ${ }^{\text {bd }}$ \\ and Willem K. Kegel ${ }^{\star b}$
}

In colloids with competing short-range attractions and long-range repulsions, microcrystalline gels are experimentally formed under conditions where computer simulations point to a lamellar phase as the ground state. Here, upon applying a low-frequency alternating electric field, we bring the system from an initial gel state to a columnar-like state. While molecular dynamics simulations on monodisperse colloids reveal that a columnar structure spontaneously evolves towards a lamellar phase, the columnarlike state in experiments relaxes back to the initial disordered gel state once the electric field is switched off. Similarly, a columnar phase in molecular dynamics simulations decomposes into finite-size crystalline clusters as the relative polydispersity of the colloids is around $1.0 \%$. We conclude that the experimentally observed melting of the columnar structure is driven by polydispersity. Moreover, further simulations reveal that the critical polydispersity required to destabilize a long-range ordered structure increases with the attraction range, pointing to the possibility of observing periodic structures in experiments if the attraction range is sufficiently long compared to the polydispersity of the colloids.

\section{Introduction}

Microphase separation in colloids with competing short-range attractions and long-range repulsions (SALRs) can produce finitesize equilibrium clusters. ${ }^{1-3}$ At high volume fractions, computer simulations found columnar or lamellar phases as the ground states. ${ }^{4,5}$ However, these periodically ordered states have never been observed in experiments. Instead, gel states are frequently observed. Regarding the gelation, mechanisms including jamming of clusters, ${ }^{6}$ repulsive glass of clusters, ${ }^{7}$ cluster percolation $^{8-12}$ and interrupted transition from a disordered phase to an ordered phase $\mathrm{e}^{5,13}$ have been suggested. A common feature of these mechanisms is that the disordered gel states are metastable with respect to columnar or lamellar phases. Direct evidence for this feature comes from a recent molecular dynamics (MD) simulation: a deep quench gives rise to a disordered network which, at a longer time, gradually evolves into a columnar phase. ${ }^{14}$ This scenario has so far not been observed in experiments, however. The viscosity of the solvent and the strong

\footnotetext{
${ }^{a}$ Center for Soft Condensed Matter Physics and Interdisciplinary Research, Soochow University, Suzhou, 215006, P. R. China.E-mail: zhangtianhui@suda.edu.cn

${ }^{b}$ Van't Hoff Laboratory for Physical and Colloid Chemistry, Utrecht University, Padualaan 8, 3584 CH Utrecht, The Netherlands.E-mail: w.k.kegel@uu.nl

${ }^{c}$ College of Physics, Optoelectronics and Energy, Soochow University, Suzhou, 215006, P. R. China

${ }^{d}$ South China Academy of Advanced Optoelectronics, South China Normal University, Guangzhou, 510006, P. R. China
}

$\dagger$ Electronic supplementary information (ESI) available. See DOI: $10.1039 / \mathrm{c} 4 \mathrm{sm} 02273 \mathrm{~d}$ attractive bonding between contacting particles may play an essential role in inhibiting or slowing down the evolution from a disordered to an ordered state. So far, the understanding of the formation of gel states is still far from being complete although it has been intensively studied for decades. In hard-sphere and repulsive colloidal systems, it has been shown that the polydispersity of colloidal particles has a significant consequence in inhibiting the formation of long-range ordered structures. ${ }^{15,16}$ Nevertheless, the polydispersity of colloidal particles has not been taken into account to understand the mechanism of gelation in colloids with competing interactions. Therefore, it is of great interest to understand the role of polydispersity in SALR colloids in the formation of gel states.

In this study, the role of polydispersity in SALR colloids is observed by combining experiments and molecular dynamics simulations. To rule out the gelation induced by fast quenching and strong attractive bonding, a short-range attraction of a few $k_{\mathrm{B}} T \mathrm{~s}$ is used and experimentally, the colloidal dispersion is slowly and continuously concentrated by controlled sedimentation. Under these conditions, compact crystalline clusters are found at low volume fractions. At a volume fraction of about 0.35 , the crystalline clusters associate into a gel network, giving rise to the so-called microcrystalline gel. ${ }^{17}$ The simulations by de Candia et al. indicate that the ground state under the experimental conditions for the microcrystalline gels is a lamellar structure. ${ }^{5}$ Yet, experimentally, no spontaneous evolution from the gel to long-range ordered phase occurs, although the formation of crystalline clusters indicates that rearrangements necessary for the formation of ordered 
structures occur on experimental time scales. To bring the system out of the gel state, a low frequency alternating electrical field (AEF) is applied to shear the microcrystalline gel, and columnar-like structures are produced. However, as the AEF is switched off, the columnar structures spontaneously decompose into small crystalline clusters and the microcrystalline gel reenters the system. In simulations, the columnar structures in a monodisperse system coalesce and gradually evolve into sheet-like structures, being structurally equivalent to lamellar ordering; in a polydisperse system, however, the presence of a polydispersity as small as $1.0 \%$ destabilizes the columnar structures and gives rise to a microcrystalline gel. Regarding the polydispersity of $3.0 \%$ in the experimental colloids, it follows that the melting of columnar structures observed in experiments is due to the presence of polydispersity. Finally we show that the critical polydispersity required to destabilize periodic structures strongly depends on the range of attractions in SALR colloids.

\section{Materials and methods}

In our experiments, polymethylmethacrylate (PMMA) spheres are used. By static light scattering (SLS) and scanning electron microscopy (SEM), the mean radius and the polydispersity of the PMMA spheres are determined to be $446 \mathrm{~nm}$ and 3.0\% respectively. ${ }^{18}$ For observation by confocal microscopy, the PMMA spheres are fluorescently labelled with 4-metylaminoethylmethacrylate-7-nitro-2-oxa-1,3-diazoal (NBD-MAEM) and sterically stabilized against flocculation by poly(12-hydroxystearic acid). The PMMA spheres are dispersed in a mixture of cyclohexyl bromide (CHB) and cis-decalin. In this solvent, PMMA spheres become positively charged which leads to a longrange Coulomb repulsion between them. The long-range repulsion is complemented with a short-range depletion attraction by adding non-adsorbing polystyrene polymers (molecular weight: $200000 \mathrm{~g} \mathrm{~mol}^{-1}$ ). The polymers in the solvent have a mean radius of gyration $r_{\mathrm{g}}=12.5 \mathrm{~nm}$ which determines the range of the depletion attraction. The minimum of the short-range attractive potential $U$ is determined using $U /$ $k_{\mathrm{B}} T=-0.5 \phi_{\mathrm{p}}(2+3 / \varphi)$, where $\varphi=r_{\mathrm{g}} / a$ and $\phi_{\mathrm{p}}$ is the fraction of the free volume of solution occupied by polymer coils. ${ }^{19,20}$ In this study, $\phi_{\mathrm{p}}$ in all samples is $\sim 0.10\left(\sim 4.0 \mathrm{mg} \mathrm{ml}^{-1}\right)$, corresponding to $U \sim-5.5 k_{\mathrm{B}} T$. Upon slowly increasing the colloidal volume fraction by sedimentation, microcrystalline gels are formed as described in ref. 17 and 21.

To examine the influence of polydispersity on the experimentally observed structure, we perform MD simulations. In our simulations, a rectangular size distribution as used in previous studies $^{22,23}$ is employed. The size distribution of the particles in terms of their diameter is defined by $\sigma_{i}=\sigma_{\max }-$ $(i-1) \Delta, i=1,2, \ldots, N$. Here, $\sigma_{\max }$ is the maximum particle size, $N$ is the number of particle species and $\Delta$ is the size step. The polydispersity $\delta$ is defined as the ratio between the standard deviation and the average diameter: $\delta=\sqrt{\left\langle\sigma^{2}\right\rangle-\langle\sigma\rangle^{2}} /\langle\sigma\rangle$. Given the size step, the polydispersity is then determined by $N$. The short-range attraction is modeled by a modified Lennard-
Jones potential with exponent $\alpha=100$ and the long-range electrostatic repulsion is modeled by a Yukawa potential. The total pair potential is:

$$
V_{i j}(r)=4 \varepsilon\left[\left(\frac{\sigma_{i j}}{r}\right)^{2 \alpha}-\left(\frac{\sigma_{i j}}{r}\right)^{\alpha}\right]+A_{i j} \frac{\exp (-r / \lambda)}{r / \lambda}
$$

where $\sigma_{i j}=\left(\sigma_{i}+\sigma_{j}\right) / 2$ and the diameters are scaled relative to $\sigma_{\text {max }}=1 ; \lambda=2 \sigma_{\max }$ and $A_{11}=0.2 \varepsilon$ are chosen such that the effective attraction range is comparable to the experimental case of $3.0 \%$. For particles with different sizes, the surface potential is assumed to be a constant and $A_{i j}$ is then adjusted accordingly as reported in ref. 16 . In simulations, the time is measured in the units of $\sqrt{m \sigma_{\max ^{2} / \varepsilon}}$ and the time step is $\delta t=0.002$.The pair interaction is cut off at $8 \sigma_{\max }$. The temperature $T$ is in the units of $\varepsilon / k_{\mathrm{B}} T$ and is set at $0.2 \mathrm{in}$ all simulations. Periodic boundary conditions are employed and the size of the simulation box in all three directions is larger than $16 \sigma_{\max }$. Columnar or lamellar phases are constructed as the starting configurations and then structure evolutions at different polydispersities are followed.

\section{Results and discussion}

Fig. 1a (top) presents a typical microcrystalline gel (an extensive network of crystalline clusters). The colloidal volume fraction in the microcrystalline gel is approximately 0.35 . The 2D Fourier transform (inset in Fig. 1) of the $x-y$ section exhibits no sharp intensity peaks but an isotropic interior high intensity region and an outside low density region, suggesting that the structure is globally isotropic. To examine the stability of microcrystalline gels, a low frequency AEF is applied. The frequency $f$ is $2 \mathrm{~Hz}$ and the peak-to-peak field strength $E_{\mathrm{pp}}$ is $0.15 \mathrm{~V} \mu \mathrm{m}^{-1}$. The response of the colloids induces an oscillating shear in the system. After shearing the sample for five minutes, columnar-like structures parallel to the electric field are formed (Fig. 1b, top). Correspondingly, the 2D Fourier transform (inset in Fig. 1b) exhibits an anisotropic pattern. Combined with the cross-section image of the structure in the $x-z$ plane (Fig. 1b, bottom), it is confirmed that the structures have a columnar topology. On the free energy landscape, columnar structures under the conditions of $U=-5.5 k_{\mathrm{B}} T$ and the colloidal volume fraction of $\sim 0.35$ are metastable with respect to lamellar structures. ${ }^{5,24,25}$ Therefore, it is expected that the columnar structures in our experiments will coalesce and evolve toward sheet-like structures. However, that is not what happens, the columnar structures in the experiments neither persist nor coalesce into sheet-like structures as the AEF is turned off. Instead, as Fig. 1c and d show, the columnar structures gradually decompose into smaller crystalline clusters and eventually, the columnar structures vanish and a microcrystalline gel reenters the system.

Globally similar order to disorder transitions have been observed in hard-sphere ${ }^{26}$ and repulsive colloids. ${ }^{16,23}$ In these observations, disorder is being induced by size polydispersity. Regarding SALR colloids, however, the effect of polydispersity has not been addressed so far. Recently, it was suggested ${ }^{\mathbf{1 4 , 2 7}}$ that a small polydispersity ( $>1 \%$ ) in SALR colloids may inhibit the formation of ordered structures. To unveil the role of 

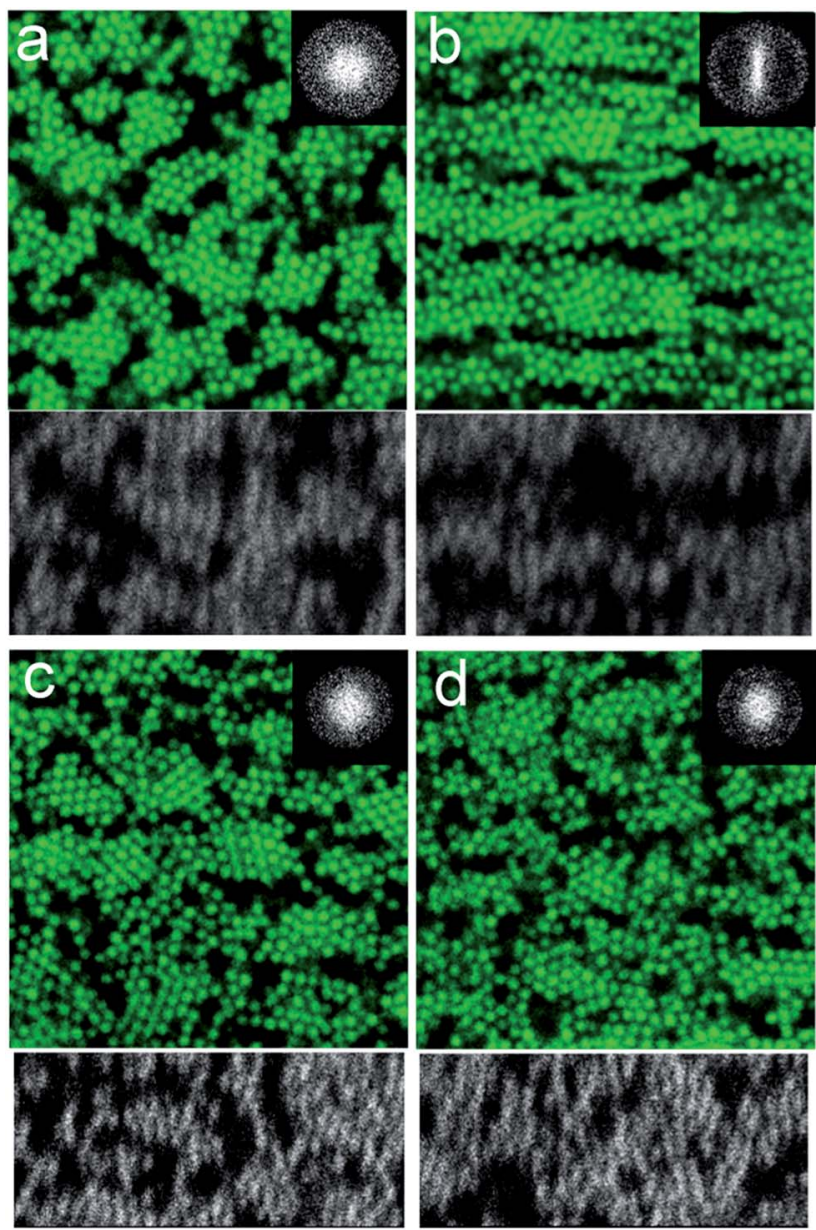

Fig. 1 Experimental observation of the structure evolution of the microcrystalline gel with a confocal microscope. (a) Microcrystalline gel on the objective plane (top). (b) Columnar structures are formed upon applying the AEF. (c) Intermediate state observed four days after the AFE is switched off. (d) Reentrant microcrystalline gel observed one week after the AFE is switched off. The gray images are the crosssection of the three-dimensional structures on the $x-z$ plane, which are reconstructed from 3D confocal scanning.

polydispersity in our experiments, MD simulations are conducted. To address the fate of a columnar phase in the presence of polydispersity, a columnar phase (Fig. S1a in the ESI $\dagger$ ) is constructed as the initial configuration. First, the stability of the columnar phase in a monodisperse system is examined and it is found that the columnar phase gradually coalesces into sheetlike structures (Fig. 2a). The sheets are not parallel to each other but the interior of the sheet-like structures is well-ordered (Fig. 2b). Since the sheet-like structures are highly analogous to lamellar structures, we conclude that the columnar phase is indeed not stable with respect to lamellar phases, in agreement with ref. 5. Now we address the question what the fate of the columnar structure is in the presence of polydispersity. Based on the study of Auer and Frenkel, ${ }^{15}$ we assume in the following simulations that the initial columnar structures are composed of sub-crystalline domains (Fig. S1a in the ESI $\dagger$ ). Particles in each sub-crystalline domain are identical in size and the sub-
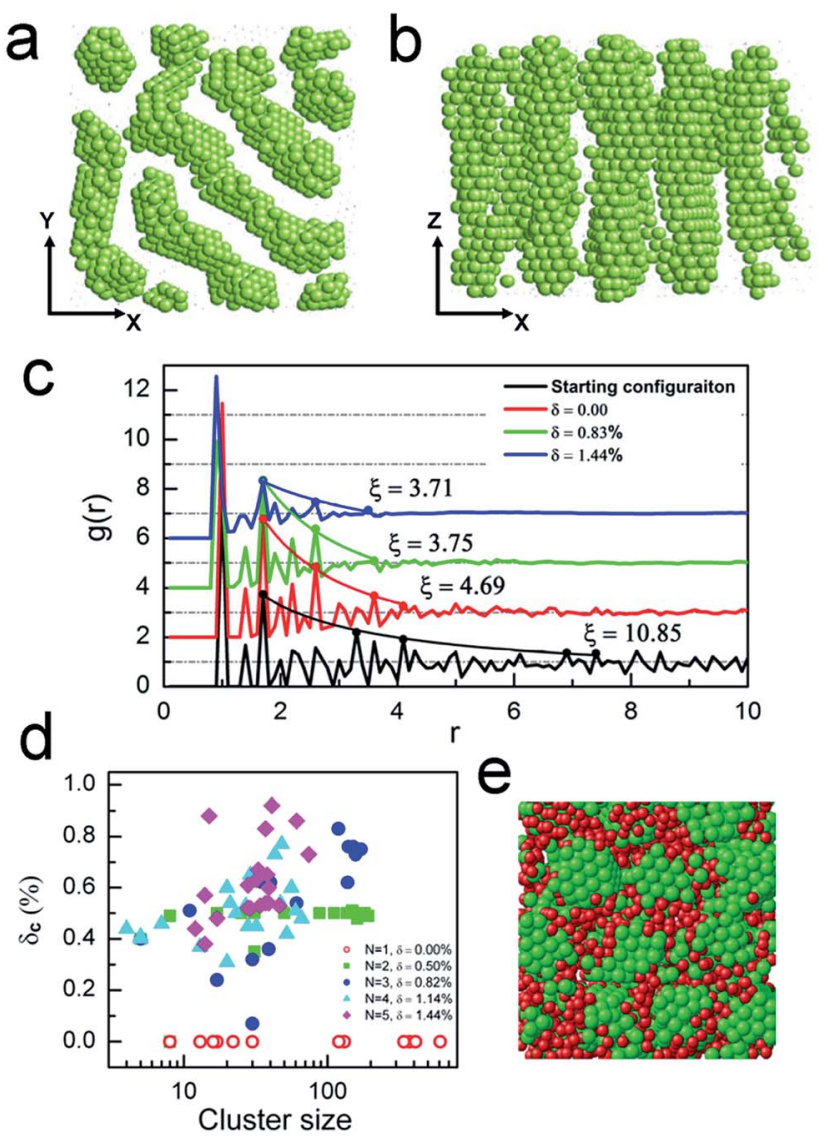

e

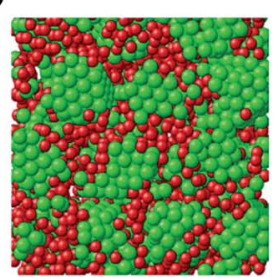

Fig. 2 Simulation results of the effect of polydispersity on the stability of columnar phases. ( $a$ and b) Sheet-like structures obtained in a monodisperse system. (a) is the top view along the $z$-direction. (b) is the side view along the $y$ direction. Particles with less than 8 bonds are not shown for a better view. (c) Radial distribution function in various structures. (d) Distribution of $\delta_{\mathrm{c}}$ which is the size polydispersity in individual crystalline clusters. (e) Microcrystalline gel observed at $\delta=1.44 \%$. Red particles: $\delta_{l}>1.0 \%$. Green particles: $\delta_{l}<1.0 \%$.

crystalline domains may be distinct from each other in terms of the size of their constituent particles. In this case, a size step of $\Delta=0.01$ is used for the rectangular distribution.

The simulation results show that upon increasing the polydispersity, the final structure obtained by simulations becomes increasingly disordered. This can be seen from the radial distribution function (RDF) shown in Fig. 2c: as a sign of crystalline order, the sharp peaks of RDF decay slowly with distance in the starting columnar phase and the height of peaks is still significant even when the distance is beyond 10; upon increasing the polydispersity, the peaks decay faster with distance. The decay superimposed on the peaks results from the finite size of crystalline structures and there exists a correlation length $\xi$ beyond which the system becomes homogeneous or disordered, i.e. $g(r)=1$ (to give a clear view, the $g(r)$ of different structures is shifted up accordingly). The decay of the peaks can be fitted by a power-law and the position where the fitting curve goes to 1.0 gives the correlation length. ${ }^{28}$ The correlation length $\xi$ offers a definite measurement of the typical size of crystalline clusters. Fig. 2c shows that increasing the polydispersity leads to smaller crystalline clusters. 
To have a better understanding of the final structures, the size and polydispersity $\delta_{\mathrm{c}}$ of crystalline clusters that survived the dynamic evolution are measured. Two particles are regarded as bonded if their center-to-center distance is less than 1.2 which is the position of the middle point of the first peak and the second peak of the radial distribution function. In previous studies, a group of bond-order parameters based on spherical harmonics were developed and widely used to measure the local crystallinity and identify the specific symmetry of the local structures. ${ }^{29,30}$ Using the local bond-order parameters, the most ordered particles will be identified as crystal-like; however, depending on the selection of the critical values of bond-order parameters, more or less surface particles will be included. In our case, it is not critical to identify the specific symmetry of local structures. Therefore, a simple criterion is used: particles with more than 8 bonds are defined as crystal-like particles. Clusters composed of crystal-like particles are identified as crystalline clusters. Fig. $2 \mathrm{~d}$ shows that $\delta_{\mathrm{c}}$ is consistently lower than $1.0 \%$ in all simulated systems. In the monodisperse system, the number of particles in the largest sheet-like cluster is up to 700. Upon increasing the polydispersity, the size of the largest crystalline clusters decreases and as the polydispersity is beyond $1.0 \%$, no crystalline structure larger than 100 exists. Given the size 140 of the starting columnar structures in simulations, it follows that column coalescence happens in the monodisperse system while columns decompose in systems with the polydispersity above $1.0 \%$. At $\delta=1.44 \%$, the final structure (Fig. 2e) is an extensive network of crystalline clusters which are randomly orientated, being highly similar to the experimentally observed microcrystalline gel. To quantify the size distribution in the final structure, a local polydispersity $\delta_{1}$ is defined. Different from the overall polydispersity $\delta, \delta_{1}$ is computed only with a central particle and its nearest neighbors (Fig. S1b in the ESI $\dagger$ ). The higher the $\delta_{1}$, the broader is the local size distribution. In Fig. 2e, particles with a local polydispersity $\delta_{1}<1.0 \%$ are colored with green while red particles represent the ones with a local polydispersity $\delta_{1}>1.0 \%$. Clearly, the crystalline domains are generally formed by particles with $\delta_{1}<1.0 \%$ while disordered regions are associated with particles of $\delta_{1}>1.0 \%$. Based on the above simulations, we put forward that the presence of a polydispersity of $3.0 \%$ in our experiments will destabilize a columnar phase. Then, it is plausible to attribute the experimentally observed melting of the columnar structures to the relatively high polydispersity.

The simulation results presented in Fig. 2 indicate that a lamellar phase in a monodisperse system is more stable with respect to a columnar phase. The mechanism is that in colloids with competing short-range attractions and long-range repulsions (SALR), the short-range attraction drives the system to form compact structures, while the long-range repulsion prefers dispersed states. As a result of the competition, or 'compromise', small clusters are stable at low overall colloidal volume fractions. ${ }^{1-3}$ At higher volume fractions, packing constraints lead to modulated structures, in particular columnar and lamellar phases. ${ }^{4,5}$ However, Fig. 2 shows that the presence of a polydispersity of around $1.0 \%$ can effectively inhibit the evolution from columnar phases to lamellar phases. To directly study the fate of a lamellar phase in the presence of polydispersity, a lamellar structure consisting of parallel close-packed bilayers is taken as the initial configuration. The particles in the lamellar bilayers are arranged with a face-centered-cubic structure and the lattice constant is the distance corresponding to the minimum of the interaction potential between particles with a diameter of $\sigma=1$. Particles with distinct diameters are randomly distributed in all lamellae (see Fig. S2a in the ESI $\dagger$ ) and the inter-lamellar separation is modified such that the overall volume fraction is 0.35 . Our simulations indicate that the lamellar structure in the absence of polydispersity is indeed stable, being in agreement with previous studies. ${ }^{4,5}$ Since the results shown in Fig. 2 reveal that a polydispersity of around $1.0 \%$ can effectively destabilize a long-range ordered structure, the fate of a lamellar phase with a polydispersity of $1.0 \%$ is examined. In this case, a smaller size step $\Delta=0.002$ is used to reduce the effect of the size discontinuity and the number of particle species is then determined as $N=17$. As expected, the lamellar phase melts and a gel-like state containing short-range ordered structures is formed (Fig. 3a). This structure is similar to the experimentally observed microcrystalline gel.

In theory, crystalline structures are still possible in a polydisperse system if size fractionation is allowed: by fractionation, a polydisperse system can fractionate into coexisting crystals
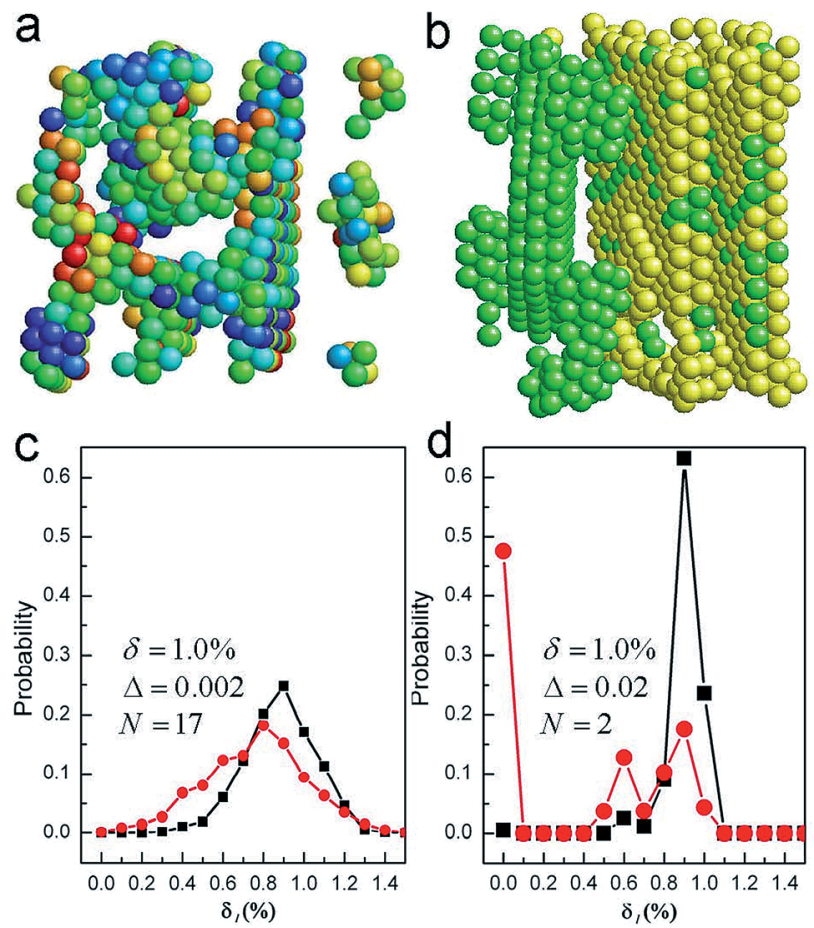

Fig. 3 Simulation results of the effect of polydispersity on lamellar phases. (a) Gel-like state obtained in a polydisperse system. The particles with bonds less than 8 are not presented. Particles are colored by size. (b) In the binary system with a polydispersity of $1.0 \%$, fractionation occurs and gives rise to single-component lamellae. (c) and (d) present the probability distribution of local polydispersity $\delta_{l}$ in the final structures obtained at a polydispersity of $1.0 \%$. (c) In the polydisperse system. (D) In the binary system. Black solid squares: the starting lamellar phase. Red solid circles: the end structure. 
with lower polydispersities. ${ }^{22,31}$ Therefore, a stable lamellar structure may be expected if the particles segregate into bilayers with relatively low polydispersity, i.e., much lower than the initial polydispersity of $1.0 \%$. Due to the continuous size distribution in the experiments, and the many $(N=17)$ discrete size categories in the simulation, it is plausible that such a segregated lamellar state is not reached and the system gets arrested in a 'micro-crystalline gel'. To verify this scenario, we study a binary system with $\Delta=0.02\left(\sigma_{i}=1,0.98\right.$ and $\left.i=1,2\right)$ such that the polydispersity is again $1.0 \%$. However, due to only two particle species, the kinetics of fractionation toward periodic structures should become significantly faster. As expected, the lamellae melt first and subsequently, fractionation and crystallization occur in the molten and disordered regions. Stable bonds are reformed between equally sized particles, giving rise to single-component crystalline nuclei that grow and ultimately form size-segregated lamellar bilayers, i.e. bilayers containing equally sized particles as shown in Fig. $3 \mathrm{~b}$.

In the binary system (Fig. 3d), the $\delta_{1}$ distribution in the initial lamellar phase has a single peak at $\delta_{1}=0.9$. This indicates that most particles in the initial situation have at least one nearest neighbor which is different in size. In the final lamellar structure, however, the vast majority of particles has $\delta_{1}=0$, indicating significant segregation. As can be seen in Fig. 3c, in the system with the more continuous distribution there is also a significant segregation, although not (almost) complete as in the binary system. Clearly, the local polydispersity in the system with the more continuous size distribution is significantly lowered, while at the same time the system becomes more disordered. It follows that in the presence of a polydispersity of $\sim 1.0 \%$, lamellar structures are unstable and tend to melt. In the binary system, melting is followed by fractionation. Singlecomponent lamellae are formed and the polydispersity in the newly formed lamellar bilayers is essentially zero. This observation is consistent with the theoretical study of Sollich et al. ${ }^{22,31}$

The critical polydispersity to melt a crystal in a hard-sphere system is at least $4.0 \%$ (ref. 26) while in our simulations, a polydispersity of $1.0 \%$ is high enough to destabilize a crystalline structure. Obviously, the critical polydispersity strongly depends on the nature of the colloidal interactions. In SALR colloids, a small fluctuation in the distance between particles may lead to a significant interaction change - possibly from attractive to repulsive. We expect that the shorter the range of attraction, the more sensitive the SALR colloids may become to polydispersity. To verify this scenario, an additional computer simulation is performed with the binary system of $1.0 \%$. In this simulation, the exponent $\alpha$ of the modified Lennard-Jones potential in eqn (1) is set at 20 such that the range of attraction is around $20 \%$ of a colloidal diameter. In this case, all simulations start with the same configuration as shown in Fig. S2b† and the results are shown in Fig. 4. As expected, the starting lamellar phase becomes stable and no melting or fractionation of the hybrid lamellae occurs, being in contrast to the observations presented in Fig. 3b. For $\alpha=20$, the lamellar phase is stable even as the polydispersity is increased to $4.2 \%$. As a consequence, the average pair potential energy remains constant in the simulations for both polydispersities of $1.0 \%$

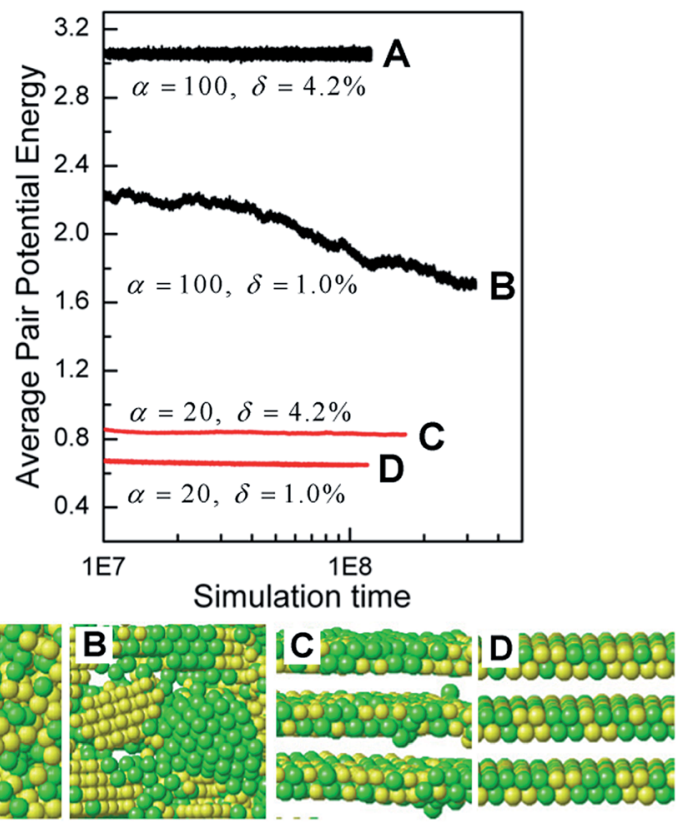

Fig. 4 Simulation results of the effect of the attraction range on the stability of a lamellar phase. The system becomes more sensitive to polydispersity as the attraction range becomes shorter in terms of the structure stability and the average pair potential energy. Given the range of attractions, the average pair potential energy becomes higher as the polydispersity is increased. For the short-range attraction $(\alpha=100)$, the average pair potential energy decreases significantly over time in the simulations due to the size fractionation and structure reorganization at the polydispersity of $1.0 \%$. Snapshot B shows the proceeding fractionation. As the polydispersity is increased to $4.2 \%$, the system is deeply arrested in a disordered state and the average pair potential energy exhibits no significant change during the simulations. For the long-range attraction ( $\alpha=20$ ), the lamellar phase is stable both in the structure (Snapshot D) and in the average pair potential energy during the simulations although the lamellar sheets become slightly distorted (Snapshot C) at a high polydispersity of $4.2 \%$.

and $4.2 \%$ (Fig. 4, Curves D and C); for a higher polydispersity, the average pair potential energy is higher. Similarly, for $\alpha=100$ as the polydispersity is increased to $4.2 \%$, the average pair potential energy also becomes higher than that of polydispersity $1.0 \%$. Moreover, Fig. 4 shows that with the same increase of polydispersity, the increase of the average pair potential energy for the short-range attraction $(\alpha=100)$ is significantly larger than that of the long-range attraction $(\alpha=20)$. For the shortrange attraction, as the polydispersity is increased to $4.2 \%$, the lamellar phase becomes unstable and melts quickly in the simulations. As Snapshot A in Fig. 4 (bottom) shows, the fate of the system is clearly a disordered state where no size fractionation and structure reorganization occur before it is frozen. As a consequence, the average pair potential energy does not change in the following simulations (Fig. 4, Curve A). In comparison, size fractionation and structure reorganization clearly do occur after melting in the case of a polydispersity of $1.0 \%$ (Snapshot B in Fig. 4) and accompanying the structure evolution, the average pair potential energy decreases significantly over the time during the simulations (Fig. 4, Curve B). 
In summary, in SALR colloids with a polydispersity, initially formed long-range ordered structures are unstable and tend to segregate, leading to a 'microcrystalline gel'. These microcrystalline gels have a much lower local polydispersity than in the original structure. Our computer simulations indicate that ultimately, microcrystalline gels should evolve towards segregated long-ranged periodic structures. However, because of the large number of metastable states, and the requirement of global fractionation, this evolution becomes extremely slow and the systems can be regarded as being arrested. The experimentally observed melting of columnar structures can be attributed to the presence of a polydispersity significantly higher than $1.0 \%$. In SALR colloids, the critical polydispersity above which periodic structures are unstable is small and strongly depends on the range of attractions; increasing the range of attractions beyond the relative polydispersity of the colloids may ultimately lead to stable periodic structures.

\section{Acknowledgements}

T. H. Zhang and W. K. Kegel acknowledge financial support from Netherlands Institute for Space Research (SRON). T. H. Zhang acknowledges the financial support from National Natural Science Foundation of China No. 11374218. W. Tian acknowledges the financial support from National Natural Science Foundation of China No. 91027040 and No. 21104056. Alfons van Blaaderen and Hanumantha Rao Vutukuri are thanked for initial discussions and preliminary experiments.

\section{References}

1 J. Groenewold and W. K. Kegel, J. Phys. Chem. B, 2001, 105, 11702.

2 J. Groenewold and W. K. Kegel, J. Phys.: Condens. Matter, 2004, 16, S4877-S4886.

3 A. Stradner, H. Sedgwick, F. Cardinaux, W. C. K. Poon, S. U. Egelhaaf and P. Schurtenberger, Nature, 2004, 432, 492.

4 M. Tarzia and A. Coniglio, Phys. Rev. Lett., 2006, 96, 075702.

5 A. de Candia, E. D. Gado, A. Fierro, N. Sator, M. Tarzia and A. Coniglio, Phys. Rev. E: Stat., Nonlinear, Soft Matter Phys., 2006, 74, 010403(R).

6 P. N. Segrè, V. Prasad, A. B. Schofield and D. A. Weitz, Phys. Rev. Lett., 2001, 86, 6042.

7 F. Sciortino, S. Mossa, E. Zaccarelli and P. Tartaglia, Phys. Rev. Lett., 2004, 93, 055701.

8 H. Sedgwick, S. U. Egelhaaf and W. C. K. Poon, J. Phys.: Condens. Matter, 2004, 16, S4913.
9 M. M. van Schooneveld, V. W. A. de Villeneuve, R. P. A. Dullens, D. G. A. L. Aarts, M. E. Leunissen and W. K. Kegel, J. Phys. Chem. B, 2009, 113, 4560.

10 R. Sanchez and P. Bartlett, J. Phys.: Condens. Matter, 2005, 17, S3551.

11 A. I. Campbell, V. J. Anderson, J. S. van Duijneveldt and P. Bartlett, Phys. Rev. Lett., 2005, 94, 208301.

12 J. C. F. Toledano, F. Sciortino and E. Zaccarelli, Soft Matter, 2009, 5, 2390.

13 M. Tarzia and A. Coniglio, Phys. Rev. Lett., 2006, 96, 075702.

14 A. Coniglio, A. de Candia and A. Fierro, Mol. Phys., 2011, 109, 2981-2987.

15 S. Auer and D. Frenkel, Nature, 2001, 413, 711-713.

16 M. N. van der Linden, A. van Blaaderen and M. Dijkstra, J. Chem. Phys., 2013, 138, 114903-114910.

17 T. H. Zhang, J. Groenewold and W. K. Kegel, Phys. Chem. Chem. Phys., 2009, 11, 10827.

18 R. P. A. Dullens, M. Claesson, D. Derks, A. van Blaaderen and W. K. Kegel, Langmuir, 2003, 19, 5963-5966.

19 H. N. W. Lekkerkerker, W. C. K. Poon, P. N. Pusey, A. Stroobants and P. B. Warren, Europhys. Lett., 1992, 20, 559.

20 S. Asakura and F. Oosawa, J. Polym. Sci., Polym. Symp., 1958, 33, 183.

21 T. H. Zhang, J. Klok, R. H. Tromp, J. Groenewold and W. K. Kegel, Soft Matter, 2012, 8, 667.

22 P. Sollich and N. B. Wilding, Soft Matter, 2011, 7, 4472-4484. 23 L. A. Fernández, V. Martín-Mayor and P. Verrocchio, Phys. Rev. Lett., 2007, 98, 085702.

24 M. Tarzia and A. Coniglio, Phys. Rev. E: Stat., Nonlinear, Soft Matter Phys., 2007, 75, 011410.

25 P. Charbonneau and D. R. Reichman, Phys. Rev. E: Stat., Nonlinear, Soft Matter Phys., 2007, 75, 050401(R).

26 P. Chaudhuri, S. Karmakar, C. Dasgupta, H. R. Krishnamurthy and A. K. Sood, Phys. Rev. Lett., 2005, 95, 248301.

27 A. Coniglio, Personal communication.

28 A. Nakano, L. Bi, R. K. Kalia and P. Vashishta, Phys. Rev. B: Condens. Matter Mater. Phys., 1994, 49, 9441.

29 P.-R. ten Wolde, M. J. Ruiz-Montero and D. Frenkel, Faraday Discuss., 1996, 104, 93-110.

30 P. J. Steinhardt, D. R. Nelson and M. Ronchetti, Phys. Rev. B: Condens. Matter Mater. Phys., 1983, 28, 784.

31 M. Fasolo and P. Sollich, Phys. Rev. E: Stat., Nonlinear, Soft Matter Phys., 2004, 70, 041410. 